\title{
Transarterial (Chemo-)Embolization and Lipiodolization for Hepatic Haemangioma
}

\author{
Alicia Furumaya ${ }^{1} \cdot$ Belle V. van Rosmalen ${ }^{1,7}$ (D) R. Bart Takkenberg ${ }^{2} \cdot$ \\ Otto M. van Delden ${ }^{3}$ Cornelis H. C. Dejong ${ }^{4,5}$ • Joanne Verheij6 • \\ Thomas M. van Gulik ${ }^{1}$
}

Received: 16 October 2018/ Accepted: 16 January 2019/Published online: 19 February 2019

(C) The Author(s) 2019

\begin{abstract}
Background Transarterial (chemo-)embolization/lipiodolization (TAE/TAL) might be an attractive minimally invasive alternative to surgery in the treatment of symptomatic hepatic haemangioma. This review assesses the efficacy and safety of TAE/TAL as primary treatment for symptomatic hepatic haemangioma.

Methods A systematic search of the literature was performed by two reviewers following the PRISMA guidelines. Cohort studies and case reports were identified;
\end{abstract}

Joanne Verheij and Thomas M. van Gulik shared senior authorship. Alicia Furumaya and Belle V. van Rosmalen shared first authorship.

Electronic supplementary material The online version of this article (https://doi.org/10.1007/s00270-019-02169-x) contains supplementary material, which is available to authorized users.

Belle V. van Rosmalen

b.v.vanrosmalen@amc.uva.nl

1 Department of Surgery, Amsterdam UMC, University of Amsterdam, Amsterdam, The Netherlands

2 Department of Gastroenterology and Hepatology, Amsterdam UMC, University of Amsterdam, Amsterdam, The Netherlands

3 Department of Interventional Radiology, Amsterdam UMC, University of Amsterdam, Amsterdam, The Netherlands

4 Department of Surgery, Maastricht University Medical Centre, Maastricht, The Netherlands

5 Department of Surgery, Uniklinikum Aachen, Aachen, Germany

6 Department of Pathology, Amsterdam UMC, University of Amsterdam, Amsterdam, The Netherlands

7 Department of Surgery, Amsterdam UMC, University of Amsterdam, Meibergdreef 9, Room G4-108, 1105AZ Amsterdam, The Netherlands outcomes of cohort studies were reported. The primary efficacy outcome was tumour size before and after TAE/ TAL. Improvement of symptoms and quality of life (QoL) were secondary outcomes; the primary safety outcome was complications. The Downs and Black statement was used for quality assessment.

Results Eighteen cohort studies were identified, including 1284 patients. TAE/TAL led to a decrease in tumour size in $1100 / 1223(89.9 \%)$ patients and to improvement or disappearance of symptoms in 1080/1096 (98.5\%) patients. A significant decrease in tumour size from $9.79 \pm 0.79 \mathrm{~cm}$ to $4.00 \pm 1.36 \mathrm{~cm}(p<0.001)$ was shown. Grade 3 complications occurred in $37 / 1284(2.9 \%)$ patients. Surgical treatment was necessary in 35/1284 (2.7\%) of patients.

Conclusion TAE/TAL appears to be a promising and safe treatment to decrease tumour size of hepatic haemangioma. The technique might also provide partial relief of symptoms, although no randomized clinical trials or prospective studies using validated QoL questionnaires are available. TAE/TAL may be considered as a viable alternative to resection.

Keywords Hepatic haemangioma - Benign liver tumour - Lipidolization - Transarterial chemoembolization · Embolization · Interventional radiology

\section{Introduction}

Haemangioma, occurring predominantly in middle-aged women, is the most common benign liver tumour [1-5]. The reported incidence varies from 0.4 to $20 \%$ in the general population $[1,2,6,7]$. In most cases, no symptoms 
are reported and the tumour is discovered incidentally on imaging for unrelated pathologies [8-13]. No treatment or follow-up imaging is required in such cases [11, 14-20].

Treatment indications are symptomatic (i.e. abdominal pain, nausea and early satiety) haemangioma, progressive growth and high risk of bleeding. Frequently, an open or laparoscopic surgical approach is used for resection of symptomatic haemangioma $[10,18,21-25]$. Common complications of surgery are blood loss, bile leakage, ileus and wound infection with a reported morbidity of $13-21 \%$ and a mortality of $0-2 \%[8,26-30]$.

Haemangiomas are composed of endothelial cells from the hepatic artery $[8,12,31]$, suggesting a place for vessel occlusive therapies such as transarterial embolization (TAE). Chemotherapeutic agents may be added to TAE with the intention to achieve additional volume reduction through the prevention of blood vessel (re)growth; this is called transarterial chemoembolization (TACE). Chemotherapeutic agents can also be administered without vessel occlusive agents, for example, in combination with ethiodized oil (lipiodolization) [32, 33]. TAE has been used as a treatment for hepatocellular adenoma, with promising results [34]. In recent years, transarterial (chemo-)embolization and lipiodolization (TAE/TAL) have been suggested to be effective in the treatment of hepatic haemangioma [13, 31, 35], either preoperatively to reduce intraoperative blood loss [13] or as definitive treatment $[8,31,35]$. Due to the non-invasive nature of the procedure, cosmetic outcomes are favourable. However, TAE/ TAL does entail exposure to radiation. No comprehensive evaluation of TAE/TAL in the literature has been performed to our knowledge.

The aim of this review is to assess the safety and efficacy of TAE/TAL as definitive treatment for haemangioma of the liver.

\section{Methods}

\section{Study Identification}

The databases MEDLINE (PubMed) and Embase were systematically searched with the aid of a clinical librarian (F.v.E.). Articles were screened by title and abstract by two independent reviewers (A.F. and B.V.v.R); inconsistent judgement was resolved by consensus. The PRISMA guidelines were followed throughout the entire process [36]. The search strategy and full study protocol are included in supplementary file 1 .

\section{Inclusion and Exclusion Criteria}

Reviews, studies not in English, French or German, studies including patients under the age of eighteen or patients with extra-hepatic haemangioma, concomitant malignancies, inherited or syndromal disease or ruptured haemangioma were excluded. If full texts were unavailable, they were purchased. Studies reporting on patients with hepatic haemangioma who had undergone TAE/TAL were included. Cohort studies were included, with no limit of a minimal number of patients; data of case reports were reported separately in tables S3-S6.

\section{Data Collection and Definitions}

Data were extracted using a standardized data extraction form. The following characteristics were collected: study design, age, sex, number of patients included, number of tumours per patient and follow-up duration, embolic agent, number of procedures, prophylactic and supportive care and imaging modalities used before and after treatment. Technical failure, as defined in the supplementary appendix, was also recorded. Indications for treatment were recorded. (Please note that the presence of symptoms before and after treatment was not the same parameter as indication for treatment.) Symptoms were recorded as the reason for treatment only if explicitly stated. Tumour size was recorded as the reason for treatment only if explicitly stated (i.e. because of damage to surrounding structures, vena cava inferior syndrome or dietary problems caused by pressure on the stomach).

The aim of this review was to determine the efficacy and safety of TAE/TAL treatment. The primary outcome measure for efficacy was tumour size. The following outcome parameters were assessed: tumour size before and after the procedure (in $\mathrm{cm}$ ), changes in tumour size according to the RECIST criteria [37], number of TAE/ TAL sessions, number of patients undergoing surgery after TAE/TAL and reason to proceed to surgery. The RECIST criteria are commonly used for malignant liver tumours but were applied for benign disease. If available, data on volumetric analysis were also extracted [38].

Secondary outcomes regarding efficacy of TAE/TAL were (relief of) symptoms and quality of life (QoL). Therefore, the presence and type of symptoms at presentation and after TAE/TAL were recorded. If the extent of symptom relief was not reported, this was recorded as partial relief to prevent an overestimation of treatment effect.

Safety was measured by recording complications according to the Cardiovascular and Interventional Radiological Society of Europe (CIRSE) classification [39]. 
Finally, type of complication and TAE-/TACE-related mortality and overall mortality were reported.

\section{Quality Assessment}

The Downs and Black criteria were used to assess methodological quality [40]. Criteria of the Downs and Black tool are provided in Table S1.

\section{Statistical Analysis}

Percentages calculated for baseline characteristics may also include data of patients not undergoing TAE/TAL if a study did not subdivide their baseline data according to treatment method. For the outcome measures, only data of patients who underwent TAE/TAL were analysed. If data were not reported separately for patients undergoing TAE/ TAL, all patients were excluded for analysis. This led to varying denominators for each outcome measure. Patients with failed embolization/lipiodolization were included in outcome analyses, following the intention-to-treat principle.

Studies reporting their data as a change in mean tumour diameter including standard deviation (SD) were analysed by weighted average, and a paired $t$ test was performed.

\section{Results}

The original search yielded 705 articles; one other article was identified by hand-searching. Then, one copy of the articles that were identified twice, in the MEDLINE as well as in the OVID search (duplicates), was removed. After removal of these duplicates, 496 articles underwent title and abstract screening. Subsequently, 154 articles were assessed by full text. Finally, a total of 18 cohort studies were eligible for inclusion [8, 16, 31, 38, 41-52], five of which had a prospective design [31, 49, 51-53]. The full study selection process is shown in Fig. 1.

\section{Quality Assessment}

Supplementary table 1 shows the full critical appraisal of each individual study. The studies by Sun et al. [50], Ji et al. [43] and Li et al. scored highest on the assessment with the Downs and Black: 15, 14 and 13 points, respectively [31]. The studies by Cao et al. [41], Martin et al. [46], Li et al. had the lowest scores: 6, 6 and 4 points, respectively [44].

\section{Baseline Characteristics}

A total of eighteen articles including 1531 patients met the eligibility criteria. Baseline characteristics of these patients are shown in Table 1. Of these patients, 1284 underwent TAE/TAL. The majority was female, 981/1522 (64.5\%), and the weighted mean age was 43.5 years. In $302 / 1320$ $(22.9 \%)$ patients, multiple haemangiomas were identified. The number of tumours in the left, right and both lobes was $81 / 428(18.9 \%), 256 / 428(59.8 \%)$ and 91/428 (21.3\%) tumours, respectively. Comorbidity is reported in table S2. Patients were followed for a weighted mean of 46.8 months.

\section{Indications for Treatment}

The indication for treatment was clearly stated for 231/1284 patients (18.0\%) [8, 16, 38, 41-43, 45, 48-50, 53]. The main treatment indication was tumour size (104/231 patients; 45.0\%). Another reason for treatment was incapacitating symptoms (83/231 patients; $35.9 \%$ ). Multiple reasons for treatment, present in 39/231 patients (16.9\%), always included symptoms. Three studies stated that the indication for treatment was either symptoms, fear of future bleeding, patients' desire for treatment or tumour size, but did not specify how many patients underwent treatment for each indication [51, 52, 54]. Details are tabulated in Table 2.

\section{Diagnostic and Treatment Methods}

Table 3 shows an overview of diagnostic and treatment methods. Mostly, ultrasound, computed tomography and magnetic resonance imaging were applied. Biopsy was occasionally used in case of unsure diagnosis or if the study was conducted before widespread availability of CT and MRI [16, 31, 38, 48, 51]. Lipiodolization was performed with either pingyangmycin or bleomycin [8, 31, 38, 45, 47, 50, 52-54]. TAE/TACE procedures usually included the use of gelfoam [41, 43, 44, 46, 48, 49]. Use of prophylactic antibiotic therapy was reported in six studies [8, 38, 48, 49, 53, 54]; supportive treatment, usually analgesia and antiemetics, was used in twelve studies [8, 31, 38, 41, 43, 45, 48-51, 53, 54]. One study used glutathione for reasons not mentioned by the authors [31].

\section{Success Rate}

Multiple embolization/lipiodolization procedures were performed [8, 31, 38, 44, 45, 48, 53, 54] for different reasons in each study, but especially in patients with large tumours [8, 31, 38, 44, 53]. Failure of embolization/lipiodolization occurred in $4 / 1244$ patients $(0.3 \%)$ [48, 52]. 
Fig. 1 PRISMA flow chart of the study selection process

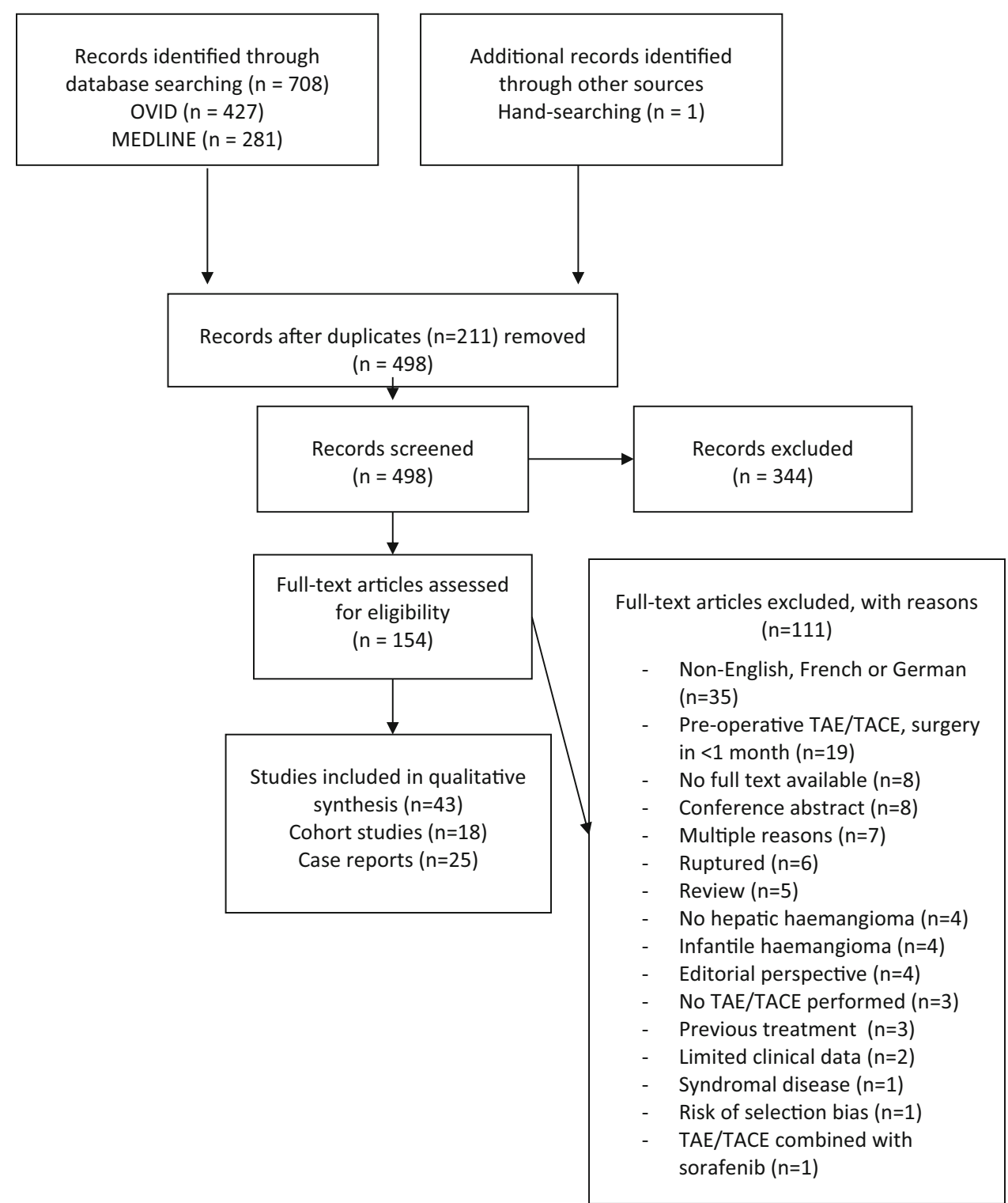

Two of these patients were followed up without further treatment, one patient underwent exploratory laparotomy with open cannulation and embolization of the tumour [48], the last patient deviation from the protocol was deemed necessary and acceptable [52]. In four articles, the length of hospital stay was reported. Bozkaya et al. [8], Kirnap et al. [53], Özden et al. and Ji et al. reported 30 h, 28 h, 24-48 h and 2.2 days of hospital stay, respectively [38, 43]. This resulted in a weighted average hospital stay of 1.5 days.

\section{Tumour Size Analysis}

Data from 1223/1284 (95.2\%) patients in fifteen studies were reported following the RECIST guidelines; tumour size was reduced in 1100 patients $(89.9 \%)$, stable in 89 patients $(7.3 \%)$. Growth of tumour was reported in 34 patients $(2.8 \%)$, all in one study [45]. The change in tumour size was measured after a weighted average of 8.6 months. In table S7, details on treatment of multiple haemangiomas are reported.

The outcomes of the eight studies (including 1047 patients) that reported tumour size in $\mathrm{cm}$ before and after embolization/lipiodolization are shown in Fig. 2. The mean tumour diameter before TAE/TAL was $9.79 \pm 0.79 \mathrm{~cm}$; after TAE/TAL, this decreased to $4.00 \pm 1.36 \mathrm{~cm}$ $(p<0.001)$. Volumetric analysis was performed in three papers. The article by Özden et al. demonstrated a reduction in median volume from 466 to $108 \mathrm{~cm}^{3}$; in the article by Bozkaya et al, the mean volume decreased from 446 to $244 \mathrm{~cm}^{3}$ [8, 38]. Kirnap et al. reported a mean volume reduction from 3716 to $746 \mathrm{~cm}^{3}$ [53]. 
Table 1 Baseline characteristics of patients included in cohort studies

\begin{tabular}{|c|c|c|c|c|c|c|}
\hline & Total/embolized & Solitary/multiple & Left/right/both & Age (years) & Female/male & Mean follow-up (months) \\
\hline Akhlaghpoor et al. [54] & $23 / 23$ & $20 / 3, \mathrm{~T}: 29$ & $9 / 20 / 0^{\mathrm{i}}$ & 46.7 & $25 / 4^{\mathrm{i}}$ & 7.5 \\
\hline Bozkaya et al. [8] & $26 / 26$ & $\mathrm{~T}: 32$ & $4 / 24 / 4$ & 49.8 & $21 / 5$ & 7.4 \\
\hline Cao et al. [41] & $14 / 14$ & NA & NA & 44.0 & $7 / 7$ & $21.0^{\mathrm{f}}$ \\
\hline Farges et al. [16] & $163 / 5$ & $108 / 55^{\mathrm{a}}$ & NA & $48.1^{\mathrm{a}}$ & $123 / 40^{\mathrm{a}}$ & $92.0^{\mathrm{a}}$ \\
\hline Firouznia et al. [42] & $20 / 20$ & $20 / 5, \mathrm{~T}: 25$ & $8 / 17 / 0$ & 46.8 & $16 / 4$ & 6.0 \\
\hline Ji et al. [43] & $15 / 15$ & $10 / 5^{\mathrm{b}}$ & $2 / 8 / 5$ & 43.5 & $9 / 6$ & 7.0 \\
\hline Kirnap et al. [53] & $17 / 17$ & $6 / 11$ & $2 / 7 / 8$ & 46.4 & $10 / 7$ & 14.5 \\
\hline Li et al. [44] & $75 / 10$ & $56 / 19^{\mathrm{a}}$ & $14 / 53 / 12^{\mathrm{a}}$ & $37.4^{\mathrm{a}}$ & $45 / 30^{\mathrm{a}}$ & 6.0 \\
\hline Li et al. [31] & $836 / 836$ & 720/116, T: 1120 & NA & 42.8 & $535 / 301$ & 52.8 \\
\hline Liu et al. [45] & $55 / 55^{\mathrm{h}}$ & NA & NA & 43.1 & $38 / 17$ & 60.0 \\
\hline Martin et al. [46] & $7 / 7$ & $2 / 5$ & NA & 40.4 & $5 / 2$ & $38.8^{\mathrm{e}}$ \\
\hline Ouyang et al. [47] & $30 / 21$ & $8 / 22$ & $4 / 13 / 13$ & $43.0^{\mathrm{a}}$ & $25 / 5^{\mathrm{a}}$ & NA \\
\hline Özden et al. [38] & $25 / 25$ & $9 / 16^{\mathrm{c}}$ & NA & 47.6 & $21 / 4$ & $14.0^{\mathrm{g}}$ \\
\hline Reading et al. [48] & $24 / 9$ & NA & NA & 45.0 & $6 / 3$ & $24.0^{\mathrm{ag}}$ \\
\hline Srivastava et al. [49] & $8 / 8$ & 7/1, T: 9 & $1 / 5 / 2$ & 47.8 & $3 / 5$ & $9.0^{\mathrm{f}}$ \\
\hline Sun et al. [50] & $27 / 27$ & $10 / 17$ & $1 / 13 / 13$ & 47.7 & $21 / 6$ & 9.6 \\
\hline Wang et al. [51] & $68 / 68$ & $42 / 26$ & $20 / 35 / 12$ & 41.0 & $45 / 23$ & $13.5^{\mathrm{f}}$ \\
\hline Zeng et al. [52] & 98/98 & NA & $16 / 61 / 21$ & 41.6 & $26 / 72$ & 12.0 \\
\hline Total & $1531 / 1284$ & 1018/302, T: 1215 & $81 / 256 / 91$ & $43.5^{\mathrm{d}}$ & $981 / 541$ & $46.8^{\mathrm{d}}$ \\
\hline
\end{tabular}

Total/embolized $=$ number of patients with haemangioma/number of patients undergoing TAE/TAL for haemangioma, Solitary/multiple $=$ number of patients with solitary haemangioma/number of patients with multiple haemangioma, $\mathrm{T}=$ total number of haemangioma, Left/ right/both = number of haemangioma in the left liver lobe/number of haemangioma in the right liver lobe/number of haemangioma in both liver lobes, Age $=$ mean age in years, NA $=$ not available

${ }^{a}$ Reported for all patients with haemangioma, not only patients undergoing TAE/TAL

${ }^{\mathrm{b}}$ Unclear method of reporting: multiple haemangiomas in one lobe are counted as single haemangioma

${ }^{\mathrm{c}}$ Includes twelve patients with $>3$ lesions

${ }^{\mathrm{d}}$ Weighted average

${ }^{\mathrm{e}}$ Two patients lost to follow-up

${ }^{\mathrm{f}}$ Follow-up reported as a range converted to a mean

${ }^{\mathrm{g}}$ Reported as a median

${ }^{\mathrm{h}}$ Eight patients excluded due to loss to follow-up

${ }^{\mathrm{i}}$ Reported for the number of lesions, not the number of patients

\section{Symptoms and Symptom Relief}

Of note, only one study used standardized tools to measure QoL or symptoms. Verbal rating scales were used by Kirnap et al. to measure the severity of pain [53], demonstrating that $9 / 23$ patients became painless after TAE/TAL. The seven patients with initial severe symptoms had all improved. In most patients, 1192/1284 (92.8\%), details on symptoms were reported. The majority, $1072 / 1192$ (89.9\%), was symptomatic at presentation. In $163 / 1152$ patients $(14.1 \%)$, a more detailed description of complaints was given.

Complaints were abdominal pain and discomfort in $65 / 163$ patients (40.0\%), bloating in 33/163 (20.2\%), abdominal distension and mass effect in 4/163 (2.5\%), multiple reasons in 56/163 (34.3\%) and other in $5 / 163$ $(3.1 \%)$.

After treatment, symptoms were recorded for 1096/1284 $(85.4 \%)$ patients. Complete resolution of symptoms occurred in 81/1096 patients (7.4\%). In 999/1096 (91.1\%), there was partial symptom relief, and in 16/1096 patients $(1.5 \%)$, persistent or recurrent symptoms were reported. Five of the patients with persistent symptoms were eventually relieved of their symptoms, two by TAE/TAL and three by measures not reported [48]. Included articles generally did not state how long after TAE/TAL symptom relief occurred. 
Table 2 Reason for treatment and outcomes in cohort studies

\begin{tabular}{|c|c|c|c|c|c|c|c|}
\hline & $\begin{array}{l}\text { Indications } \\
\text { for TAE/ } \\
\text { TAL }\end{array}$ & $\begin{array}{l}\text { Reasons (not) to perform } \\
\text { additional procedures }\end{array}$ & Tumour size reduction & $\begin{array}{l}\text { Follow- } \\
\text { up }\end{array}$ & Symptoms & S.I & Complications \\
\hline $\begin{array}{l}\text { Akhlaghpoor } \\
\text { et al. [54] }\end{array}$ & NA & Elective: reason not stated & $\mathrm{R}: 29$ & 7.5 & PD: 23 & NA & $\begin{array}{l}\text { Grade } 1 \text { (13): non- } \\
\text { target } \\
\text { embolization } \\
\text { Grade } 1 \text { (6): PES }\end{array}$ \\
\hline $\begin{array}{l}\text { Bozkaya } \\
\text { et al. [8] }\end{array}$ & $\begin{array}{l}\text { AS: } 24 \\
\text { E: } 2\end{array}$ & $\begin{array}{l}\text { Elective (26): patients unfit for } \\
\text { surgery }\end{array}$ & $\begin{array}{l}\varnothing 9.72-7.63 \\
\text { S: } 26\end{array}$ & 7.4 & SO: 26 & I: 26 & $\begin{array}{l}\text { Grade } 3(1) \text { : } \\
\text { ischaemic } \\
\text { cholecystitis } \\
\text { Grade } 1 \text { (26): PES }\end{array}$ \\
\hline $\begin{array}{l}\text { Cao et al. } \\
\text { [41] }\end{array}$ & $\mathrm{L}: 14$ & Elective: unresectable tumour & $\mathrm{R}: 8,>50 \%$ reduction & 1 & NA & D: 14 & Grade 1 (11): PES \\
\hline $\begin{array}{l}\text { Farges et al. } \\
\quad[16]\end{array}$ & AS: $16^{\mathrm{a}}$ & $\begin{array}{l}\text { Elective: directed at } \\
\text { intratumoural arterial fistula } \\
(1), \text { reason not stated (4) }\end{array}$ & $\mathrm{R}: 7, \mathrm{G}: 9^{\mathrm{a}}$ & NA & PD: $87^{\mathrm{a}}$ & $\begin{array}{l}\text { D: } 1 \\
\text { P: } 4\end{array}$ & None \\
\hline $\begin{array}{l}\text { Firouznia } \\
\quad \text { et al. [42] }\end{array}$ & $\begin{array}{l}\text { AS: } 15 \\
\text { E: } 5\end{array}$ & Elective: reason not stated & S: $20, \varnothing 9.70-8.90$ & 6 & NA & I: 20 & Grade 2 (1): PES \\
\hline Ji et al. [43] & $\begin{array}{l}\text { AS: } 3 \\
\text { E: } 4 \\
\text { M: } 8\end{array}$ & $\begin{array}{l}\text { Elective: refusal to surgery. } \\
\text { RFA after TAE/TAL }\end{array}$ & $\mathrm{R}: 15, \varnothing 13.0-7.1$ & 1 & PD: 15 & $\begin{array}{l}\text { D: } 10 \\
\text { I: } 1 \\
\text { NA: } 4\end{array}$ & $\begin{array}{l}\text { Grade } 3(8) \text { : } \\
\text { postprocedural } \\
\text { pain }\end{array}$ \\
\hline $\begin{array}{l}\text { Kirnap et al. } \\
\text { [53] }\end{array}$ & M: 17 & Elective: reason not stated & $\begin{array}{l}\mathrm{R}: 17, \varnothing 14.7-7.6, \\
\text { mean volume } \\
3716 \mathrm{~cm}^{3} \text { to } 746 \mathrm{~cm}^{3}\end{array}$ & 12 & $\begin{array}{l}\text { PD: } 5 \\
\text { B: } 11 \\
\text { O: } 1\end{array}$ & I: 17 & $\begin{array}{l}\text { Grade } 3 \text { (1): low } \\
\text { haemoglobin } \\
\text { Grade } 1 \text { (7): PES }\end{array}$ \\
\hline Li et al. [44] & NA & Elective: experimental & S: 10 & 6.0 & & NA & $\begin{array}{l}\text { Grade 3: lasting } \\
\text { pain }^{\mathrm{e}}\end{array}$ \\
\hline Li et al. [31] & NA & Elective: patients choice & $\mathrm{R}: 836, \varnothing 9.6-3.6$ & NA & SO: 836 & I: 836 & $\begin{array}{l}\text { Grade } 3 \text { (2): hepatic } \\
\quad \text { abscess } \\
\text { Grade 1: PES }\end{array}$ \\
\hline Liu et al. [45] & L: 55 & $\begin{array}{l}\text { Elective (24): reason not stated } \\
\text { Pre-operative (31): enlarging } \\
\text { tumour (29) or severe } \\
\text { complication (2) }\end{array}$ & $\begin{array}{l}\text { S: } 19 \\
\text { G: } 34\end{array}$ & NA & $\begin{array}{l}\text { PD: } 8 \\
\text { B: } 20 \\
\text { M: } 2 \\
\text { A: } 25\end{array}$ & NA & $\begin{array}{l}\text { Grade } 3 \text { (2): biloma } \\
\text { Grade } 3 \text { (2): hepatic } \\
\text { abscess }\end{array}$ \\
\hline $\begin{array}{l}\text { Martin et al. } \\
{[46]}\end{array}$ & NA & Elective: reason not stated & $\mathrm{R}: 2, \mathrm{~S}: 2^{\mathrm{b}}$ & $21^{\mathrm{b}}$ & $\begin{array}{l}\text { PD: } 3 \\
\text { B: } 2 \\
\text { ME: } 1 \\
\text { A: } 1\end{array}$ & $\begin{array}{l}\text { D: } 1 \\
\text { I: } 2 \\
\text { P: } 2 \\
\text { NA: } \\
2^{\text {b }}\end{array}$ & $\begin{array}{l}\text { Grade } 1(3) \text { : } \\
\text { postembolization } \\
\text { pain }\end{array}$ \\
\hline $\begin{array}{l}\text { Ouyang et al. } \\
{[47]}\end{array}$ & NA & $\begin{array}{l}\text { Elective: directed at } \\
\text { arteriovenous shunt }\end{array}$ & NA & NA & $\begin{array}{l}\text { PD: } 6 \\
\text { A: } 24^{\mathrm{a}}\end{array}$ & NA & NA \\
\hline $\begin{array}{l}\text { Özden et al. } \\
{[38]}\end{array}$ & $\begin{array}{l}\text { AS: } 15 \\
\text { E: } 3 \\
\text { M: } 7\end{array}$ & Elective: reason not stated & $\begin{array}{l}\text { Median volume } \\
466 \mathrm{~cm}^{3} \text { to } 108 \mathrm{~cm}^{3}\end{array}$ & $8^{\mathrm{d}}$ & A: 3 & $\begin{array}{l}\text { I: } 17 \\
\text { P: } 5 \\
\text { NA: } 3\end{array}$ & $\begin{array}{l}\text { Grade } 3(1) \text { : } \\
\text { transient allergic } \\
\text { rash } \\
\text { Grade } 2 \text { (3): PES } \\
\text { Grade } 1 \text { (22): PES }\end{array}$ \\
\hline $\begin{array}{l}\text { Reading et al. } \\
\text { [48] }\end{array}$ & $\begin{array}{l}\text { AS: } 7 \\
\text { H: } 1 \\
\text { O: } 1\end{array}$ & Elective: reason not stated & $\mathrm{R}: 1, \mathrm{~S}: 6^{\mathrm{c}}$ & NA & $\begin{array}{l}\text { PD: } 3 \\
\text { M: } 3 \\
\text { A; } 1 \\
\text { ME: } 1 \\
\text { O: } 1\end{array}$ & $\begin{array}{l}\text { D: } 1 \\
\text { I: } 1 \\
\text { P: } 5\end{array}$ & $\begin{array}{l}\text { Grade } 3 \text { (2): hepatic } \\
\quad \text { abscesses } \\
\text { Grade } 3 \text { (6): PES }\end{array}$ \\
\hline $\begin{array}{l}\text { Srivastava } \\
\text { et al. [49] }\end{array}$ & M: 7 & $\begin{array}{l}\text { Elective: experimental } \\
\text { Pre-operative (1): persistent } \\
\text { symptoms }\end{array}$ & $\mathrm{R}: 1, \mathrm{~S}: 4, \varnothing 9.28-8.62$ & 9 & $\begin{array}{l}\text { PD: } 6 \\
\text { O: } 2\end{array}$ & D: 8 & Grade 1 (8): PES \\
\hline
\end{tabular}


Table 2 continued

\begin{tabular}{|c|c|c|c|c|c|c|c|}
\hline & $\begin{array}{l}\text { Indications } \\
\text { for TAE/ } \\
\text { TAL }\end{array}$ & $\begin{array}{l}\text { Reasons (not) to perform } \\
\text { additional procedures }\end{array}$ & Tumour size reduction & $\begin{array}{l}\text { Follow- } \\
\text { up }\end{array}$ & Symptoms & S.I & Complications \\
\hline $\begin{array}{l}\text { Sun et al. } \\
\text { [50] }\end{array}$ & $\begin{array}{l}\text { AS: } 3 \\
\text { E: } 21 \\
\text { O: } 3\end{array}$ & $\begin{array}{l}\text { Elective: reason not stated. } \\
\text { Pre-operative }(1) \text { : surgery } \\
\text { possible after TAE/TAL, } \\
3 \text { weeks post-TAE/TAL }\end{array}$ & $\mathrm{R}: 27, \varnothing$ 11.24-7.60 & 6 & $\begin{array}{l}\text { PD: } 2 \\
\text { O: } 1 \\
\text { A: } 24\end{array}$ & $\begin{array}{r}\text { I: } 4 \\
\text { NA: } \\
23\end{array}$ & $\begin{array}{l}\text { Grade } 3 \text { (12): mild } \\
\text { fever } \\
\text { Grade } 1 \text { (6): PES }\end{array}$ \\
\hline $\begin{array}{l}\text { Wang et al. } \\
\text { [51] }\end{array}$ & NA & Elective: experimental & $\begin{array}{l}\text { S: } 2, \mathrm{R}: 66,30 \text { of which } \\
\quad \text { with }>50 \% \\
\text { reduction }\end{array}$ & NA & $\begin{array}{l}\text { SO: } 47 \\
\text { A: } 21\end{array}$ & I: 68 & $\begin{array}{l}\text { Grade 1: } \\
\text { haematoma at } \\
\text { puncture site } \\
\text { Grade 1: } \mathrm{PES}^{\mathrm{e}}\end{array}$ \\
\hline $\begin{array}{c}\text { Zeng et al. } \\
{[52]}\end{array}$ & NA & $\begin{array}{l}\text { Elective: patient's choice. } \\
\text { Pre-operative (2): continued } \\
\text { pain }\end{array}$ & R: $98, \varnothing 9.70-3.00$ & 12 & $\begin{array}{l}\text { M: } 53 \\
\text { A: } 45\end{array}$ & $\begin{array}{l}\text { D: } 46 \\
\text { I: } 7 \\
\text { NA: } \\
45\end{array}$ & Grade 1 (83): PES \\
\hline Total & $\begin{array}{l}\text { AS: } 83 \\
\text { L: } 69 \\
\text { E: } 35 \\
\text { M: } 39 \\
\text { O: } 4 \\
\text { H: } 1\end{array}$ & $\begin{array}{l}\text { Pre-operative (35): enlarging } \\
\text { tumour (29), unfit for surgery } \\
\text { (26), continued symptoms } \\
\text { (3), severe complication (2) } \\
\text { or surgery possible (1) }\end{array}$ & $\begin{array}{l}\text { R: } 1100 \\
\text { S: } 89 \\
\text { G: } 34\end{array}$ & $8,6^{\mathrm{f}}$ & $\begin{array}{l}\text { SO: } 909 \\
\text { A: } 120 \\
\text { PD: } 65 \\
\text { M: } 56 \\
\text { B: } 33 \\
\text { ME: } 4 \\
\text { O: } 5\end{array}$ & $\begin{array}{l}\text { D: } 81 \\
\text { I: } 999 \\
\text { P: } 16\end{array}$ & $\begin{array}{l}\text { Grade } 3: 37 \\
\text { Grade 2: } 4 \\
\text { Grade 1: } 185\end{array}$ \\
\hline
\end{tabular}

Follow-up = timing of follow-up imaging in months, Symptoms = symptoms before TAE/TAL, SI = symptomatic improvement, AS = in order to alleviate symptoms, $\mathrm{E}=$ enlarging tumour, $\mathrm{L}=$ large tumour, $\mathrm{H}=$ haemorrhage, high bleeding risk, $\mathrm{O}=$ other, $\varnothing=$ mean diameter in $\mathrm{cm}$ before TAE/TAL - mean diameter after TAE/TAL. RECIST Criteria: $\mathrm{CD}=$ Complete disappearance of the tumour, $\mathrm{R}=$ reduction $(>30 \%)$ decrease in tumour size, $\mathrm{S}=$ stable $(<30 \%)$ decrease and $(<20 \%)$ increase in tumour size, $\mathrm{G}=$ growth $(>20 \%)$ increase in tumour size., $\mathrm{PD}=$ pain or discomfort, $\mathrm{B}=$ bloating or abdominal distension, $\mathrm{M}=$ multiple, $\mathrm{ME}=$ mass effect including dyspepsia and palpable masses, $\mathrm{SO}=$ symptoms not otherwise specified, $\mathrm{A}=$ asymptomatic, $\mathrm{D}=$ disappearance, $\mathrm{I}=$ improvement, $\mathrm{P}=$ persistent/recurrent/stable symptoms, $\mathrm{PES}=$ postembolization syndrome, $\mathrm{NA}=$ not available

${ }^{a}$ Reported for all patients with haemangioma, not only patients undergoing TAE/TAL. Excluded from analysis

${ }^{\mathrm{b}}$ Two patients lost to follow-up, one patient no imaging follow-up

${ }^{c}$ Includes patient treated with open cannulation

${ }^{\mathrm{d}}$ Median

${ }^{\mathrm{e}}$ Not reported how many patient suffered from these complications

${ }^{\mathrm{f}}$ Weighted average

\section{Additional Procedures}

After TAE/TAL, 35/1284 patients (2.7\%) underwent surgery (Table 2) [45, 50]. Ji et al. used radiofrequency ablation (RFA) on month after transarterial embolization as part of the study protocol. As tumour size was measured after TAE/TAL but before RFA, this study was included in the analysis of tumour size [43].

\section{Complications}

Complications occurred in 226/1284 (17.6\%) patients. Postembolization syndrome was the main complication, occurring in 179/1284 patients (13.9\%). Grade 3 complications were reported in 37/1284 patients (2.9\%), mainly postprocedural pain [43], mild fever [50] and hepatic abscesses. Grade 3 complications and their management are reported in table S8. No mortality was reported. 
Table 3 Diagnostic and treatment methods used in cohort studies

\begin{tabular}{|c|c|c|c|c|c|}
\hline & $\begin{array}{l}\text { Diagnostic methods before } \\
\text { TAE/TAL }\end{array}$ & $\begin{array}{l}\text { Diagnostic } \\
\text { methods after } \\
\text { TAE/TAL }\end{array}$ & TAE/TAL material & $\begin{array}{l}\text { Nr. } \\
\text { TAE/ } \\
\text { TAL }\end{array}$ & Pharmacotherapeutics \\
\hline $\begin{array}{l}\text { Akhlaghpoor } \\
\text { et al. [54] }\end{array}$ & US, CT or MRI & CT & $\begin{array}{l}\text { Bleomycin }(30-45 \mathrm{IU} \text { in } 5 \mathrm{cc} \\
\text { saline) and lipiodol }(7-15 \mathrm{cc})\end{array}$ & 3 & $\begin{array}{l}\mathrm{P}: \text { type not reported } \\
\mathrm{S}: \text { antiemetics, gastric } \\
\text { protection medications }\end{array}$ \\
\hline $\begin{array}{l}\text { Bozkaya } \\
\text { et al. [8] }\end{array}$ & $\mathrm{CT}$ or MRI & CT or MRI & $\begin{array}{l}\text { Bleomycin }(15 \mathrm{mg} \text { in } 5 \mathrm{~mL} \text { saline, } \\
\text { max. } 30 \mathrm{mg}) \text { and lipiodol } \\
(10 \mathrm{~mL}, \max .20 \mathrm{mg})\end{array}$ & 4 & $\begin{array}{l}\text { P: type not reported } \\
\text { S: sedation (pre), analgesia } \\
\text { (pre and post) and } \\
\text { antiemetics (post) }\end{array}$ \\
\hline $\begin{array}{l}\text { Cao et al. } \\
\text { [41] }\end{array}$ & US, CT or MRI & CT & $\begin{array}{l}\text { Bleomycin }(16-32 \mathrm{mg}) \text { and lipiodol } \\
(10-15 \mathrm{~mL}) \text { and gelatin sponge }\end{array}$ & NA & $\mathrm{S}$ : lidocaine $50 \mathrm{mg}$ \\
\hline $\begin{array}{l}\text { Farges et al. } \\
\text { [16] }\end{array}$ & $\begin{array}{l}\text { US (153), CT (78), MRI (38), } \\
\text { angiography (76) or } \\
\text { diagnostic exploratory } \\
\text { laparotomy }(9)^{\mathrm{a}}\end{array}$ & NA & NA & NA & NA \\
\hline $\begin{array}{l}\text { Firouznia } \\
\quad \text { et al. [42] }\end{array}$ & US, CT or MRI & $\begin{array}{l}\text { US (17) or CT } \\
\text { (3) }\end{array}$ & $\begin{array}{l}\text { Polyvinyl alcohol particles } \\
\quad(300-400 \mu \mathrm{m})\end{array}$ & 0 & NA \\
\hline Ji et al. [43] & NA & CT or MRI & $\begin{array}{l}\text { Pingyangmycin ( } 8 \mathrm{mg} \text { in } 2 \mathrm{~mL} 5 \% \\
\text { glucose) and lipiodol }(20 \mathrm{~mL}) \\
\text { and gelatin sponge particles } \\
(1-2 \mathrm{~mm})\end{array}$ & NA & S: sedation and analgesia \\
\hline $\begin{array}{l}\text { Kirnap et al. } \\
\text { [53] }\end{array}$ & US (11), CT (6) or MRI & CT & $\begin{array}{l}\text { Bleomycin }(15 \mathrm{mg} \text { in } 5 \mathrm{~mL} \text { saline }) \\
\text { and lipiodol }(10 \mathrm{~mL})\end{array}$ & 5 & $\begin{array}{l}\mathrm{P} \text { : cephazolin } 1 \mathrm{~g} \text { i.v. } \\
\text { S: analgesics, sedatives }\end{array}$ \\
\hline Li et al. [44] & $\begin{array}{l}\text { US (75), CT (66), angiography } \\
\text { (34), pathological diagnosis } \\
(21), \text { ECT }(20)^{\mathrm{a}}\end{array}$ & $\begin{array}{l}\text { US, CT or } \\
\text { scintigraphy }\end{array}$ & $\begin{array}{l}\text { Gelfoam }(100 \mathrm{mg}) \text { and lipiodol } \\
\quad(8-16 \mathrm{~mL})\end{array}$ & T: $36^{\mathrm{e}}$ & NA \\
\hline Li et al. [31] & CT or MRI & CT & $\begin{array}{l}\text { Pingyangmycin (24 mg in } 5 \mathrm{~mL} 1 \% \\
\text { lidocaine) and lipiodol }(10 \mathrm{~mL})\end{array}$ & 130 & $\begin{array}{l}\mathrm{S}: 2 \% \text { lidocaine } 5-10 \mathrm{~mL}, \\
\text { analgesia, antiemetics, } \\
\text { reduced glutathione }\end{array}$ \\
\hline Liu et al. [45] & CT or MRI & US, CT & Pingyangmycin and lipiodol & $17^{\mathrm{d}}$ & S: local anaesthesia \\
\hline $\begin{array}{l}\text { Martin et al. } \\
\text { [46] }\end{array}$ & $\begin{array}{l}\text { US (4), CT (1) or scintigraphy } \\
\text { (2) }\end{array}$ & $\begin{array}{l}\text { US (3), CT (1), } \\
\text { scintigraphy } \\
(1), \\
\text { angiography } \\
(1)^{\mathrm{b}}\end{array}$ & $\begin{array}{l}50 \% \text { isobutyl-2-cyanoacrylate and } \\
\text { lipiodol (4 patients) or Gelatin } \\
\text { sponge and thrombase ( } 3 \\
\text { patients) }\end{array}$ & NA & NA \\
\hline $\begin{array}{l}\text { Ouyang et al. } \\
\text { [47] }\end{array}$ & US, CT or angiography & NA & Bleomycin and lipiodol & NA & NA \\
\hline $\begin{array}{l}\text { Özden et al. } \\
\text { [38] }\end{array}$ & MRI (25), biopsy (1) & $\mathrm{CT}$ or MRI & $\begin{array}{l}\text { Bleomycin }(15 \mathrm{mg} \text { in } 5 \mathrm{~mL} \text { non- } \\
\text { ionic contrast agent) and lipiodol } \\
(10 \mathrm{~mL})\end{array}$ & $8^{\mathrm{c}}$ & $\begin{array}{l}\text { P: ampicillin, sulbactam } \\
\text { and amoxicillin- } \\
\text { clavulanate (pre and post) } \\
\text { S: analgesics, antiemetics } \\
\text { (pre and post) }\end{array}$ \\
\hline $\begin{array}{l}\text { Reading et al. } \\
\text { [48] }\end{array}$ & $\begin{array}{l}\text { US (24), angiography (22) or } \\
\text { biopsy }(12)^{\mathrm{a}}\end{array}$ & Angiography & $\begin{array}{l}\text { Gelfoam and sodium iothalamate } \\
\text { and } 50 \% \text { dextrose }\end{array}$ & $2^{\mathrm{d}}$ & $\begin{array}{l}\text { P aminoglycosides and } \\
\text { cephalosporins combined } \\
\text { with metronidazole (pre } \\
\text { and post) } \\
\text { S: analgesia (post) }\end{array}$ \\
\hline $\begin{array}{l}\text { Srivastava } \\
\text { et al. [49] }\end{array}$ & US, CT or MRI & US or CT & $\begin{array}{l}\text { Polyvinyl alcohol particles and/or } \\
\text { gelfoam and fibred steel coils }\end{array}$ & 0 & $\begin{array}{l}\text { P: type not reported (post) } \\
\text { S: analgesia (pre and post), } \\
\text { sedation (pre), antiemetics } \\
\text { (post) }\end{array}$ \\
\hline
\end{tabular}


Table 3 continued

\begin{tabular}{|c|c|c|c|c|c|}
\hline & $\begin{array}{l}\text { Diagnostic methods before } \\
\text { TAE/TAL }\end{array}$ & $\begin{array}{l}\text { Diagnostic } \\
\text { methods after } \\
\text { TAE/TAL }\end{array}$ & TAE/TAL material & $\begin{array}{l}\text { Nr. } \\
\text { TAE/ } \\
\text { TAL }\end{array}$ & Pharmacotherapeutics \\
\hline $\begin{array}{l}\text { Sun et al. } \\
\text { [50] }\end{array}$ & US, CT or MRI & NA & $\begin{array}{l}\text { Pingyangmycin ( } 8-16 \mathrm{mg} \text { in } 2 \mathrm{~mL} \\
5 \% \text { glucose solution) and lipiodol }\end{array}$ & NA & S: rehydration \\
\hline $\begin{array}{l}\text { Wang et al. } \\
\text { [51] }\end{array}$ & $\begin{array}{l}\text { US, CT, angiography or } \\
\text { pathological examinations }\end{array}$ & CT & $\begin{array}{l}\text { Pingyangmycin }(4-16 \mathrm{mg}) \text { and } \\
\text { super liquefaction iodipin } \\
\text { (5-20 mL), biological } \\
\text { microspheres }(300-500 \mu \mathrm{m} \text {, } \\
1-5 \mathrm{~mL})\end{array}$ & NA & $\mathrm{S}: 1 \%$ lidocaine $2-5 \mathrm{~mL}$ \\
\hline $\begin{array}{l}\text { Zeng et al. } \\
\text { [52] }\end{array}$ & US or CT & $\begin{array}{l}\mathrm{CT} \text {, chest } \\
\text { photography }\end{array}$ & $\begin{array}{l}\text { Pingyangmycin (8-24 } \mathrm{mg} \text { in } \\
2-10 \mathrm{~mL} \text { lopamiro } 300 \text { and } \\
\text { lipiodol }\end{array}$ & 0 & NA \\
\hline
\end{tabular}

Nr. TAE/TAL $=$ number of patients needing more than one TAE/TAL session, $\mathrm{T}=$ total number of TAE/TAL procedures performed, $\mathrm{P}=$ prophylactic, $\mathrm{S}=$ supportive, $\mathrm{ECT}=$ emission computed tomography (e.g. $\mathrm{PET}$ or SPECT), NA = not available

${ }^{\mathrm{a}}$ Reported for all patients with haemangioma, not only patients undergoing TAE/TAL

${ }^{\mathrm{b}}$ Two patients lost to follow-up

${ }^{\mathrm{c}}$ Includes one patient who underwent three TAE/TAL procedures

${ }^{\mathrm{d}}$ Includes two patients who underwent three TAE/TAL procedures

${ }^{\mathrm{e}}$ Including percutaneous embolization

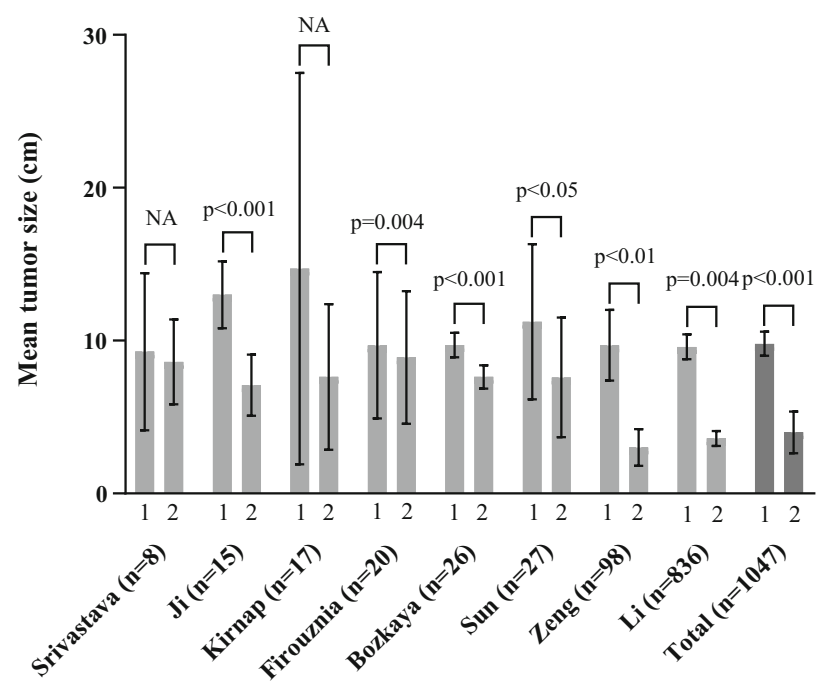

Fig. 2 Tumour size pre- and post-TAE/TACE

\section{Discussion}

TAE/TACE led to a mean reduction in size from 9.79 to $4.00 \mathrm{~cm}$. Tumour size decreased in $89.9 \%$ of patients. Surgery was not required in $97.3 \%$ of patients. TAE/TAL appeared to be an effective method to diminish or resolve symptoms; in 999 patients (91.1\%), TAE/TAL improved symptoms, and in 81 patients (7.4\%), symptoms completely disappeared. Patients were followed for a weighted mean of 46.8 months. In 37 patients $(2.9 \%)$, grade 3 complications occurred and no mortality was reported after TAE/TAL.
In current guidelines, TAE/TAL is not yet recommended as an alternative to surgery and is only considered to manage Kasabach-Merritt syndrome [25]. As this review shows promising results in regard to tumour size reduction and partial symptom relief, the role of TAE/TAL in the management of hepatic haemangioma might be reconsidered. The largest study on surgical treatment for hepatic haemangioma showed that surgery might provide symptomatic improvement of $44-87 \%$ of patients after a median follow-up of 2.8 years [55]. In comparison, in this review, partial or complete relief was present in $98.5 \%$ of patients after TAE/TAL after a median follow-up of 4 years. The occurrence of the three major morbidities, i.e. post-operative bleeding, bile leakage and liver failure was $1.8 \%, 5.1 \%$ and $1,6 \%$, respectively. In contrast, the total number of grade 3 complications after TAE/TAL was 2.9\%. After TAE/TAL, no mortality was reported compared to $0.2 \%$ mortality in the surgical study.

The largest study (836 patients) used glutathione as supportive treatment [31]. In humans, the only described indication of antioxidant use is in case of acetaminophen intoxication. There is no known indication for the use of glutathione in the setting of TAE/TAL. The complication rate of this prospective study was very low (2/836), which is interesting as most prospective studies tend to identify more complications than retrospective studies [56]. It is unclear whether the low rate of complications is associated with the administration of glutathione, the used embolization material (i.e. pingyangmycin and lipiodol) or perhaps the study size. A high volume of patients in an experienced centre is associated with better outcomes [57, 58]. 
Limitations of this review should be taken into account. Firstly, 35 articles, including a few large cohort studies, were excluded because these studies were not in English, French or German. Secondly, some of the included studies used parametric tests, while it was not clear whether the data were normally distributed, for example, the studies by Kirnap et al., Srivastava et al. and Sun et al. failed the socalled "95\% range check" [49, 50, 59]. However, these data were taken into analysis as these were good quality studies based on the quality assessment. Finally, retrospective studies are often flawed by reporting bias. This might have led to an overestimation of the effects of TAE/ TAL.

Future research should determine which treatment method is superior: transarterial (chemo-)embolization or lipiodolization. No further predictive factors of treatment success could be identified, due to the heterogeneity of studies. Ideally, TAE/TAL should be compared to conservative management and surgery. Moreover, QoL deserves a prominent role in future research because almost all patients undergoing treatment have symptoms. QoL might be evaluated by, for example, the EORTC QLQ-C30 questionnaire.

\section{Conclusion}

When treatment is indicated for liver haemangiomas (e.g. large tumour size and/or symptoms), TAE/TAL appears to be effective to decrease tumour size. It may provide relief of symptoms, however, no large prospective studies using QoL questionnaires are available. In this review, TAE/TAL was considered a safe treatment method of liver haemangiomas TAE/TAL potentially serves as a viable alternative to resection.

\section{Acknowledgments Faridi van Etten, Susan van Dieren.}

Author Contributions BVvR, AF, TMvG were involved in conceptualization, $\mathrm{BVvR}, \mathrm{AF}$ were involved in data curation, $\mathrm{BVvR}, \mathrm{AF}$ were involved in formal analysis, $\mathrm{BVvR}, \mathrm{AF}$ were involved in investigation, $\mathrm{BVvR}, \mathrm{AF}$ were involved in methodology, $\mathrm{BVvR}$, $\mathrm{TMvG}$ were involved in project administration, $\mathrm{BVvR}, \mathrm{TMvG}$ were involved in supervision, $\mathrm{BVvR}, \mathrm{AF}, \mathrm{JV}, \mathrm{TMvG}$ wrote the original draft and BVvR, AF, RBT, OMvD, CHCD, JV, TMvG reviewed and edited.

\section{Compliance with Ethical Standards}

Conflict of interest The authors declare that they have no conflict of interest.

Human and Animals Rights This article does not contain any studies with human participants or animals performed by any of the authors.
Informed Consent For this type of study informed consent is not required.

Consent for Publication For this type of study consent for publication is not required.

Open Access This article is distributed under the terms of the Creative Commons Attribution 4.0 International License (http:// creativecommons.org/licenses/by/4.0/), which permits unrestricted use, distribution, and reproduction in any medium, provided you give appropriate credit to the original author(s) and the source, provide a link to the Creative Commons license, and indicate if changes were made.

\section{References}

1. Ishak KG, Rabin L. Benign tumors of the liver. Med Clin North Am. 1975;59(4):995-1013.

2. Karhunen PJ. Benign hepatic tumours and tumour like conditions in men. J Clin Pathol. 1986;39(2):183-8.

3. Schwartz SI, Husser WC. Cavernous hemangioma of the liver. A single institution report of 16 resections. Ann Surg. 1987;205(5):456-65.

4. Trotter JF, Everson GT. Benign focal lesions of the liver. Clin Liver Dis. 2001;5(1):17-42.

5. Liu X, Yang Z, Tan H, Xu L, Liu L, Huang J, et al. Patient age affects the growth of liver haemangioma. HPB (Oxford). 2018;20(1):64-8.

6. Gandolfi L, Leo P, Solmi L, Vitelli E, Verros G, Colecchia A. Natural history of hepatic haemangiomas: clinical and ultrasound study. Gut. 1991;32(6):677-80.

7. Gilon D, Slater PE, Benbassat J. Can decision analysis help in the management of giant hemangioma of the liver? J Clin Gastroenterol. 1991;13(3):255-8.

8. Bozkaya H, Cinar C, Besir FH, Parildar M, Oran I. Minimally invasive treatment of giant haemangiomas of the liver: embolisation with bleomycin. Cardiovasc Intervent Radiol. 2014;37(1):101-7.

9. Bajenaru N, Balaban V, Savulescu F, Campeanu I, Patrascu T. Hepatic hemangioma-review. J Med Life. 2015;8(Spec Issue):4-11.

10. Hoekstra LT, Bieze M, Erdogan D, Roelofs JJ, Beuers UH, van Gulik TM. Management of giant liver hemangiomas: an update. Expert Rev Gastroenterol Hepatol. 2013;7(3):263-8.

11. Stankiewicz R, Kobryn K, Patkowski W, Krawczyk M. Management of giant hepatic hemangioma in atypical localization; report of a case and literature review. Pol Przegl Chir. 2015;87(3):139-42.

12. Vagefi PA, Klein I, Gelb B, Hameed B, Moff SL, Simko JP, et al. Emergent orthotopic liver transplantation for hemorrhage from a giant cavernous hepatic hemangioma: case report and review. J Gastrointest Surg. 2011;15(1):209-14.

13. Vassiou K, Rountas H, Liakou P, Arvanitis D, Fezoulidis I, Tepetes K. Embolization of a giant hepatic hemangioma prior to urgent liver resection. Case report and review of the literature. Cardiovasc Intervent Radiol. 2007;30(4):800-2.

14. Ng WW, Cheung YS, Lee KF, Wong J, Yu SC, Lee PS, et al. Is regular follow-up scan for giant liver haemangioma necessary? Hong Kong Med J. 2007;13(5):353-8.

15. Yamagata M, Kanematsu $T$, Matsumata $T$, Utsunomiya T, Ikeda Y, Sugimachi K. Management of haemangioma of the liver: comparison of results between surgery and observation. $\mathrm{Br} \mathrm{J}$ Surg. 1991;78(10):1223-5. 
16. Farges O, Daradkeh S, Bismuth H. Cavernous hemangiomas of the liver: are there any indications for resection? World J Surg. 1995;19(1):19-24.

17. Okano H, Shiraki K, Inoue H, Ito T, Yamanaka T, Deguchi M, et al. Natural course of cavernous hepatic hemangioma. Oncol Rep. 2001;8(2):411-4.

18. Pietrabissa A, Giulianotti P, Campatelli A, Di Candio G, Farina F, Signori S, et al. Management and follow-up of 78 giant haemangiomas of the liver. Br J Surg. 1996;83(7):915-8.

19. Tait N, Richardson AJ, Muguti G, Little JM. Hepatic cavernous haemangioma: a 10 year review. ANZ J Surg. 1992;62(7):521-4.

20. Trastek VF, van Heerden JA, Sheedy PF, Adson MA. Cavernous hemangiomas of the liver: resect or observe? Am J Surg. 1983;145(1):49-53.

21. Mezhir JJ, Fourman LT, Do RK, Denton B, Allen PJ, D’Angelica MI, et al. Changes in the management of benign liver tumours: an analysis of 285 patients. HPB (Oxford). 2013;15(2):156-63.

22. Herman P, Costa MLV, Machado MAC, Pugliese V, D'Albuquerque LAC, Machado MCC, et al. Management of hepatic hemangiomas: a 14-year experience. J Gastrointest Surg. 2005;9(6):853-9.

23. Yoon SS, Charny CK, Fong Y, Jarnagin WR, Schwartz LH, Blumgart LH, et al. Diagnosis, management, and outcomes of 115 patients with hepatic hemangioma. J Am Coll Surg. 2003;197(3):392-402.

24. Terkivatan T, de Wilt JW, de Man RA, et al. Indications and long-term outcome of treatment for benign hepatic tumors: a critical appraisal. Arch Surg. 2001;136(9):1033-8.

25. European Association for the Study of the Liver. EASL clinical practice guidelines on the management of benign liver tumours. J Hepatol. 2016;65(2):386-98.

26. Martinez-Gonzalez MN, Mondragon-Sanchez R, MondragonSanchez A, Gomez-Gomez E, Garduno-Lopez AL, Bernal-Maldonado R, et al. Cavernous hemangioma of the liver and hepatic hemangiomatosis Indications and results of the surgical resection. Rev Gastroenterol Mex. 2003;68(4):277-82.

27. Özden İ, Emre A, Alper A, Tunaci M, Acarli K, Bilge O, et al. Long-term results of surgery for liver hemangiomas. Arch Surg. 2000;135(8):978-81.

28. Ho HY, Wu TH, Yu MC, Lee WC, Chao TC, Chen MF. Surgical management of giant hepatic hemangiomas: complications and review of the literature. Chang Gung Med J. 2012;35(1):70-8.

29. Brouwers MA, Peeters PM, de Jong KP, Haagsma EB, Klompmaker IJ, Bijleveld CM, et al. Surgical treatment of giant haemangioma of the liver. Br J Surg. 1997;84(3):314-6.

30. Duxbury MS, Garden OJ. Giant haemangioma of the liver: observation or resection? Dig Surg. 2010;27(1):7-11.

31. Li Y, Jia Y, Li S, Wang W, Wang Z, Wang Y, et al. Transarterial chemoembolization of giant liver haemangioma: a multi-center study with 836 cases. Cell Biochem Biophys. 2015;73(2):469-72.

32. Brown DB, Gould JE, Gervais DA, Goldberg SN, Murthy R, Millward SF, et al. Transcatheter therapy for hepatic malignancy: standardization of terminology and reporting criteria. J Vasc Interv Radiol. 2009;20(7 Suppl):S425-34.

33. Bruix J, Sala M, Llovet JM. Chemoembolization for hepatocellular carcinoma. Gastroenterology. 2004;127(5 Suppl 1):S179-88.

34. van Rosmalen BV, Coelen RJS, Bieze M, van Delden OM, Verheij J, Dejong CHC, et al. Systematic review of transarterial embolization for hepatocellular adenomas. $\mathrm{Br} \quad \mathrm{J}$ Surg. 2017;104(7):823-35.

35. Bozkaya H, Cinar C, Ünalp ÖV, Parildar M, Oran I. Unusual treatment of Kasabach-Merritt syndrome secondary to hepatic hemangioma: embolization with bleomycin. Wien Klin Wochenschr. 2015;127(11-12):488-90.
36. Moher D, Liberati A, Tetzlaff J, Altman DG, The PG. Preferred reporting items for systematic reviews and meta-analyses: the prisma statement. PLoS Med. 2009;6(7):e1000097.

37. Eisenhauer EA, Therasse P, Bogaerts J, Schwartz LH, Sargent D, Ford R, et al. New response evaluation criteria in solid tumours: revised recist guideline (version 1.1). Eur $\mathrm{J}$ Cancer. 2009;45(2):228-47.

38. Özden İ, Poyanlı A, Önal Y, Demir AA, Hoş G, Acunaş B. Superselective transarterial chemoembolization as an alternative to surgery in symptomatic/enlarging liver hemangiomas. World $\mathbf{J}$ Surg. 2017;41(11):2796-803.

39. Filippiadis DK, Binkert C, Pellerin O, Hoffmann RT, Krajina A, Pereira PL. Cirse quality assurance document and standards for classification of complications: the cirse classification system. Cardiovasc Intervent Radiol. 2017;40(8):1141-6.

40. Downs SH, Black N. The feasibility of creating a checklist for the assessment of the methodological quality both of randomised and non-randomised studies of health care interventions. J Epidemiol Community Health. 1998;52(6):377-84.

41. Cao X, He N, Sun J, Wang S, Ji X, Wang J, et al. Interventional treatment of huge hepatic cavernous hemangioma. Chin Med J (Engl). 2000;113(10):927-9.

42. Firouznia K, Ghanaati H, Alavian SM, Nassiri Toosi M, Ebrahimi Daryani N, Jalali AH, et al. Management of liver hemangioma using trans-catheter arterial embolization. Hepat Mon. 2014;14(12):e25788.

43. Ji J, Gao J, Zhao L, Tu J, Song J, Sun W. Computed tomographyguided radiofrequency ablation following transcatheter arterial embolization in treatment of large hepatic hemangiomas. Medicine (Baltimore). 2016;95(15):e3402.

44. Li GW, Zhao ZR, Li BS, Liu XG, Wang ZL, Liu QF. Source of blood supply and embolization treatment in cavernous hemangioma and sclerosis of the liver. World $\mathrm{J}$ Gastroenterol. 1997;3(3):147-9.

45. Liu X, Yang Z, Tan H, Huang J, Xu L, Liu L, et al. Long-term result of transcatheter arterial embolization for liver hemangioma. Medicine (Baltimore). 2017;96(49):e9029.

46. Martin B, Roche A, Radice L, Aguilar K, Kraiem C. does arterial embolization have a role in the treatment of cavernous hemangioma of the liver in adults? Presse Med. 1986;15(23):1073-6.

47. Ouyang Y, Ouyang XH, Yu M, Gu SB. Frequency of arteriovenous shunts in hepatic cavernous hemangiomas in adults as seen on selective arteriography and postembolization radiography. Cardiovasc Intervent Radiol. 2001;24(3):161-7.

48. Reading NG, Forbes A, Nunnerley HB, Williams R. Hepatic haemangioma: a critical review of diagnosis and management. Q J Med. 1988;67(253):431-45.

49. Srivastava DN, Gandhi D, Seith A, Pande GK, Sahni P. Transcatheter arterial embolization in the treatment of symptomatic cavernous hemangiomas of the liver: a prospective study. Abdom Imaging. 2001;26(5):510-4.

50. Sun JH, Nie CH, Zhang YL, Zhou GH, Ai J, Zhou TY, et al. Transcatheter arterial embolization alone for giant hepatic hemangioma. PLoS ONE. 2015;10(8):e0135158.

51. Wang L, Deng C, Li J, Huang X, Fan J, Wang X, et al. Application of a new triple sequential embolization method in treatment of hepatic hemangioma. Int $J$ Clin Exp Med. 2016;9(2):5255-63.

52. Zeng Q, Li Y, Chen Y, Ouyang Y, He X, Zhang H. Gigantic cavernous hemangioma of the liver treated by intra-arterial embolization with pingyangmycin-lipiodol emulsion: a multicenter study. Cardiovasc Intervent Radiol. 2004;27(5):481-5.

53. Kirnap M, Boyvat F, Boyacioglu S, Hilmioglu F, Moray G, Haberal M. The effect of bleomycin embolization on symptomatic improvement and hemangioma size among patients with giant liver hemangiomas. Int J Surg. 2018;12:12-6. 
54. Akhlaghpoor S, Torkian P, Golzarian J. Transarterial bleomycinlipiodol embolization (b/le) for symptomatic giant hepatic hemangioma. Cardiovasc Intervent Radiol. 2018;41(11):1674-82.

55. Sakamoto Y, Kokudo N, Watadani T, Shibahara J, Yamamoto M, Yamaue H. Proposal of size-based surgical indication criteria for liver hemangioma based on a nationwide survey in japan. J Hepatobiliary Pancreat Sci. 2017;24(7):417-25.

56. Campbell PG, Malone J, Yadla S, Chitale R, Nasser R, Maltenfort MG, et al. Comparison of icd-9-based, retrospective, and prospective assessments of perioperative complications: assessment of accuracy in reporting. $J$ Neurosurg Spine. 2011;14(1):16-22.

57. Gooiker GA, van Gijn W, Wouters MW, Post PN, van de Velde CJ, Tollenaar RA. Systematic review and meta-analysis of the volume-outcome relationship in pancreatic surgery. Br J Surg. 2011;98(4):485-94.

58. Tol JA, van Gulik TM, Busch OR, Gouma DJ. Centralization of highly complex low-volume procedures in upper gastrointestinal surgery. A summary of systematic reviews and meta-analyses. Dig Surg. 2012;29(5):374-83.

59. Limpert E, Stahel WA. Problems with using the normal distribution-and ways to improve quality and efficiency of data analysis. PLoS ONE. 2011;6(7):e21403.

Publisher's Note Springer Nature remains neutral with regard to jurisdictional claims in published maps and institutional affiliations. 